\title{
ON THE CENTER OF THE GROUP OF A LINK
}

\author{
KUNIO MURASUGI
}

1. Introduction. A group $G$ is said to be finitely presented if it has a finite presentation $P$, i.e., $P$ consists of a finite number, $n$ say, of generators and a finite number, $m$ say, of defining relations. The $d e$ ficiency of $\odot, d(\odot)$, is defined as $n-m$. Then, by the deficiency, $d(G)$, of $G$ is meant the maximum of deficiencies of all finite presentations of $G$. For example, it is well known that if $G$ is a group with a single defining relation, i.e., $m=1$, then $d(G)=n-1[1$, p. 207].

Recently we proved [5] that for any group $G$ with a single defining relation, if $d(G) \geqq 2$ then the center, $C(G)$, of $G$ is trivial, and if $d(G)$ $=1$ and if $G$ is not abelian, then either $C(G)$ is trivial or infinite cyclic. This leads to the following conjecture.

Conjecture. For any finitely presented group $G$, if $d(G) \geqq 2$ then $C(G)$ is trivial, and if $d(G)=1$ and if $G$ is not abelian, then $C(G)$ is either trivial or infinite cyclic.

The purpose of this paper is to show that the conjecture is true for the group of any link in 3-sphere $S^{3}$. Namely we obtain:

THEOREM 1. If a non-abelian link group $G$ has a nontrivial center $C(G)$, then $C(G)$ is infinite cyclic.

REMARK. If $d(G) \geqq 2$, then $G$ must be a free product of two nontrivial groups. Thus $C(G)$ is trivial [9], [10].

In the case of a knot, i.e., a link of multiplicity one, Theorem 1 is an easy consequence of the fact that the center of the commutator subgroup $G^{\prime}$ of $G$ is trivial [6]. However this argument can not be generalized to the case of links as it is. To make the argument run parallel with the argument in the knot case, it is convenient to consider rather than the commutator subgroup a certain subgroup $H$, called here the canonical subgroup of $G$. The canonical subgroup might have a nontrivial center and that is the only difficult point in the proof of Theorem 1.

On the other hand, to some extent the nontriviality of the center of a link group determines the link type itself. For example, if the center of a knot group is generated by its meridian, then the knot is

Received by the editors July $11,1964$. 
trivial. ${ }^{1}$ The link with this property is uniquely determined as follows.

THEOREM 2. If the meridian of one component of a link $l$ is contained in the center of its group, then $l$ is of the following form:

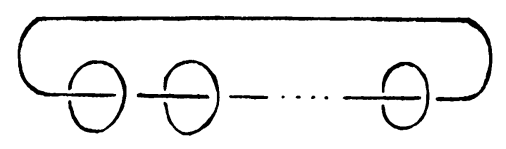

FIGURE 1

The link of this type is regarded as trivial in the sense that its Hosokawa polynomial $\nabla(t)=1[3]$.

Moreover, the following two theorems, originally proved by Neuwirth [7], are also in this direction. Their simple proofs will be seen in the last section.

TheOREM 3. If the link group $G$ is abelian, then the link $l$ is of the form:

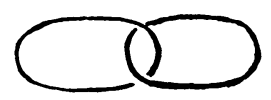

Figure 2

THEOREM 4. Let $G$ be the group of a link $l_{1} \cup l_{2}$. If $C=\left(x, y:\left[x,(x y)^{n}\right]\right){ }^{2}$ and if $x$ and $y$ are the meridians of $l_{1}$ and $l_{2}$ respectively, then $l_{1} \cup l_{2}$ is of the form (or its mirror image):

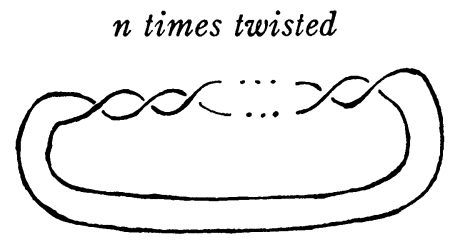

FIGURE 3

${ }^{1}$ Instead of 3-sphere, if we take an orientable compact closed 3-manifold $M$, then we can not always find a knot whose meridian generates the center of the fundamental group of the complementary of a knot in $M$. The problem of this kind has been discussed in detail by Gluck in his paper: Tangled manifolds, Ann. of Math. 76 (1962), 62-72. The author acknowledges to the referee for bringing these facts to his attention.

$2[a, b]$ denotes the commutator of $a$ and $b$. 


\section{Lemmas.}

LEMMA-1. Let $G$ be an arbitrary finitely presented group and let $H$ be a normal subgroup of $G$ such that $G / H$ is infinite cyclic. If the center of $G$ is not contained in $H$, then $H$ is finitely generated.

Proof. Since $G / H$ is abelian, $H$ contains the commutator subgroup $G^{\prime}$ of $G$. Thus we may assume without loss of generality that $G$ has a presentation $P$,

$$
\mathcal{P}=\left(t, x_{1}, x_{2}, \cdots, x_{n}: r_{1}, r_{2}, \cdots, r_{m}\right),
$$

such that $x_{i} \in H, t \notin H$ and that $r_{j}$ can be written in terms of elements in $H$. (We have to only apply Tietze's transformations on the original presentation of $G$, if necessary.)

Now since $G / H=(t:)$ and $\left\{t^{r}\right\}$ forms a Schreier representative system for $G \bmod H$, by using the well known Reidemeister-Schreier method, we obtain a presentation of $H$ as follows:

$$
\begin{array}{r}
\text { generators: } x_{i, s}=t^{s} x_{i} t^{-s}, \text { for } 1 \leqq i \leqq n,-\infty<s<\infty, \\
\text { defining relations: } r_{j, s}=r_{j}\left(\cdots, x_{i, k+s}, \cdots\right), \\
\text { for } 1 \leqq j \leqq m,-\infty<s<\infty,
\end{array}
$$

where $r_{j, 0}=r_{j}\left(\cdots, x_{i, k}, \cdots\right)$ is the result of rewriting $r_{j}\left(\cdots, x_{i}, \cdots\right)$ in terms of $x_{i, k}$.

Suppose that $C(G) \nsubseteq H$. Take an element $z \in C(G)$ such that $z \notin H$. Since $G / H \cong Z=(t:), z$ can be written in the form $z=h t^{r}$, $h \in H$ and $r$ a positive integer. Since $z$ commutes with $t^{r}$, so does $h$. Hence $t^{r p}$ commutes with $h$ for any integer $p$. Thus we see that $z^{p}=h^{p} t^{r p}$, and since $z$ commutes with each $x_{i}$, it follows that $\left[h^{p}, t\right]$ $=1$ and $\left[h^{p} t^{r p}, x_{i}\right]=1,1 \leqq i \leqq n$. Since these relations hold in $H$, we have, for any integer $p>0$,

$$
h_{0}^{p} h_{1}^{p}=1 \quad \text { and } \quad h_{0}^{p} x_{i, r p} h_{0}^{-p} x_{i, 0}^{-1}=1,
$$

and similarly, for any integer $s$,

$$
\begin{gathered}
h_{s}^{p} h_{s+1}^{-p}=1 \quad \text { and } \\
h_{s}^{p} x_{i, r p+s} \quad h_{s}^{-p} x_{i, s}^{-1}=1, \quad \text { for } 1 \leqq i \leqq n,
\end{gathered}
$$

where $h_{0}=h\left(\cdots, x_{i, k}, \cdots\right)$ is the result of rewriting $h$ in terms of $x_{i, k}$ and $h_{s}$ is the word obtained from $h_{0}$ by replacing $x_{i, k}$ by $x_{i, k+s}$.

Let $\beta_{i}$ and $\gamma_{i}$ be the minimum and maximum indices of $x_{i, k}$ which occur in $h_{0}$. If we select a positive integer $p$ so large that $-r p<\min \beta_{i}$ and $r p>\max \gamma_{i}$, then from (2.1) and (2.2) it follows that generators 
$x_{i, k}(1 \leqq i \leqq n)$ for $k \geqq r p$ or $k \leqq-r p$ are words in generators $x_{i,-r p+1}$, $\cdots, x_{i, r p-1}(1 \leqq i \leqq n)$. That is, $H$ is finitely generated. Q.E.D.

Corollary (NeUwirth [8]). If a knot group $G$ has a nontrivial center, then $G^{\prime}$ is finitely generated.

Proof. As is well known, $G$ is finitely presented and $G / G^{\prime}$ is infinite cyclic. If the center of $G$ has a nontrivial element that is contained in $G^{\prime}$, then $G^{\prime}$ would have a nontrivial center. This is a contradiction to Corollary 2 in [6].

Lemma 2. Suppose $G$ and $H$ satisfy the conditions in Lemma 1. If $d(G) \geqq 2$ and $C(H)$ is trivial, then $C(G)$ is also trivial.

Proof. Since $d(G) \geqq 2, n-1>m$. Let $\phi$ and $\psi$ be the natural homomorphisms from a free group $F=\left(t, x_{1}, \cdots, x_{n-1}:\right)$ onto $G$ and from $G$ on to $G / H \cong Z=(t:)$ respectively, such that $\psi(t)=t$ and $\psi\left(x_{i}\right)=1$, $1 \leqq i \leqq n-1$.

Suppose $C(G)$ is not trivial. Then the identity (2.1) is written as follows, setting $p=1$,

$$
h t^{r} x_{i} t^{-r-1} h x_{i}^{-1}=\prod_{j} u_{i j} r_{s_{i j}}^{e_{i j}} u_{i j}^{-1}, \quad e_{i j}= \pm 1,1 \leqq i \leqq n-1 .
$$

Differentiating both sides with respect to $x_{i}$ in (2.3) at $\psi \phi[2]$, we have the following equations in an integral group ring $J Z$ :

$$
\delta_{j, k}\left(1-t^{r}\right)=\sum_{s_{i j}=k} \alpha_{j, k}\left[\psi \phi\left(\frac{\partial r_{s_{i j}}}{\partial x_{j}}\right)\right], \quad 1 \leqq j \leqq n-1,
$$

where $\delta_{j, k}$ denotes the Kronecker's delta and $\alpha_{j, k}=\sum_{s_{i j}=k} e_{i j} u_{i j} \in J Z$. From (2.4) it follows that a matrix identity

$$
\left(1-t^{r}\right) E=\left\|\alpha_{j, k}\right\|\left\|\psi \phi\left(\frac{\partial r_{s_{i j}}}{\partial x_{j}}\right)\right\|,
$$

$E$ denoting an identity matrix. Since $n-1>m$, the right hand side must be zero, which is a contradiction. Q.E.D.

3. Proofs of theorems. Let $l$ be a link of multiplicity $\mu$ and let $G=\pi_{1}\left(S^{3}-l\right)$. Give an orientation to $l$. Let $H$ be the normal subgroup of $G$, an element of which is represented by a loop $w$ in $S^{3}-l$ such that the sum of the linking number of $w$ with each component of $l$ is zero. $H$ will be called the canonical subgroup of $G$. Since $G / H$ is infinite cyclic, the proof of Theorem 1 in [6] is valid with slight modification if $G^{\prime}$ is replaced by $H$. Thus we have

Leмma 3. (1) If $H$ is finitely generated, then it is free of rank $d$, where 
$d=2 h+\mu-1, h$ denoting the genus of an oriented link.

(2) If $H$ is not finitely generated, then either it is

(i) a nontrivial free product with amalgamation on a free group $F_{d}$ of rank $d$ :

$$
\cdots A \underset{F_{d}}{*} \underset{F_{d}}{*} A * \cdots
$$

where the amalgamations are all proper and identical, or

(ii) locally free, and a direct limit of free groups of rank d.

Proof of Theorem 1. Give an orientation to a link $l$. Let $H$ be the canonical subgroup of the link group $G$. If $C(H)$ is trivial, then $C(G)$ is either trivial or infinite cyclic, because $G / H$ is infinite cyclic. Thus we may assume that $C(H)$ is not trivial.

First we observe that $l$ consists of only two components and it spans an orientable surface of genus 0 . In fact, since $C(H)$ is not trivial, $d$ must be $=1$ for all cases in Lemma 3, which implies that $h=0$ and $\mu=2$.

Case 1 . Suppose that $H$ is finitely generated.

Since $H$ is infinite cyclic by Lemma $3, G$ is a group extension of $Z=(a:)$ by $Z=(t:)$. Thus $G=\left(t, a: t a t^{-1}=a^{\epsilon}\right), \epsilon=+1$ or -1 . If $\epsilon=1, G$ is abelian, which is excluded by the assumption. If $\epsilon=-1$, $G=\left(t, a: t^{-1}=a^{-1}\right)$, which can not be the group of a link. For, the reduced Alexander polynomial of $G$ is $1+t$. Thus this case can not happen.

Case 2. Suppose that $H$ is not finitely generated.

By Lemma $1, C(G) \subset H$, hence $C(G) \subset C(H)$. Since $C(H)$ is an infinite cyclic group according to $[4], C(G)$ is also infinite cyclic.

Thus the proof of Theorem 1 is completed.

Proof of TheORem 2. Give an orientation to a link $l$ and consider the canonical subgroup $H$ of the link group $G$. Since $H$ contains no meridian of any component of $l$ and since $C(G)$ contains the subgroup generated by a meridian $x$, it follows from Lemma 1 that $H$ must be finitely generated. Thus from Lemma 3 , we see that $H$ is a free group of rank $d$. Since we may assume that $G / H \cong Z=(x:), G$ is a group extension of $H$ by $Z=(x:)$. Thus $G$ has the following presentation $\mathcal{P}$,

$$
\odot=\left(x, a_{1}, \cdots, a_{d}:\left[x, a_{i}\right] i=1, \cdots, d\right) .
$$

Thus $l$ is a required form by Theorem D in [7].

Proof of Theorem 3. Give an orientation to $l$ and consider the canonical subgroup $H$ of $G$. Since $G$ is a finitely generated abelian group, so is $H$. Hence by Lemma 3 (1), we see that $H$ is free and abelian, i.e., $H$ is infinite cyclic. Thus $C(H)$ is not trivial. Hence the 
first half of the proof of Theorem 1 shows that the genus of $l$ is 0 and the multiplicity is two. That is, $l$ spans an orientable band $B$. Let $k$ be a core $e^{3}$ of $B$. Since $k$ is a deformation retract of $B, \pi_{1}\left(S^{3}-B\right)$ $=\pi_{1}\left(S^{3}-k\right)$. However $\pi_{1}\left(S^{3}-B\right)=H$ and $H$ is infinite cyclic. Thus $k$ is trivial by Dehn's Lemma. It is easy to show that the twisting number of $B$ must be one. Q.E.D.

Proof of Theorem 4. Give an orientation to $l$ such that $x y$ is contained in the canonical subgroup $H$ of $G$. Since $x$ is a meridian of $l_{1}$, we may assume that $G / H \cong(x:)$. Then the presentation of $H$ is given as follows:

$$
\begin{aligned}
& \text { generators: } y_{k}=x^{k} y x^{-k+1} \text {, } \\
& \text { defining relations: } r_{k}=x^{k}\left[x,(x y)^{n}\right] x^{-k} \\
& =y_{k+2}^{n} y_{k+1}^{-n}, \quad k=0, \pm 1, \cdots . \\
& \text { Thus } H=\cdots *\left(y_{0}:\right)_{\left(y_{0}^{n}=y_{1}\right)}^{*}\left(y_{1}:\right) * \cdots \text {. }
\end{aligned}
$$

Hence $C(H)=\left(y_{0}^{n}:\right)$ which implies that the genus of $l$ must be zero. Thus $l$ spans again an orientable band $B$ and the core $k$ of $B$ is unknotted, because the group of $k$ is isomorphic to $G /(x:) \cong(y:)$. It is also clear that the twisting number of $B$ must be $n$. Q.E.D.

\section{REFERENCES}

1. D. B. A. Epstein, Finite presentations of groups and 3-manifolds, Quart. J. Math. (2) 12 (1961), 205-212.

2. R. H. Fox, Free differential calculus. I, Ann. of Math. 57 (1953), 547-560.

3. - - A quick trip through knot theory, Topology of 3-manifolds, PrenticeHall, Englewood Cliffs, N. J. 1962, pp. 120-167.

4. K. A. Kurosh, The theory of groups, Vol. 2, Chelsea, New York, 1955.

5. K. Murasugi, The center of a group with a single defining relation, Math. Ann. 155 (1964), 246-251.

6. L. Neuwirth, On the algebraic determination of the genus of knots, Amer. J. Math. 82 (1960), 791-798.

7. - A note on torus knots and links determined by their groups, Duke Math. J. 28 (1961), 545-551.

8. - A remark on knot groups with a center, Proc. Amer. Math. Soc. 14 (1963), 378-379.

9. C. D. Papakyriakopoulos, On Dehn's lemma and the asphericity of knots, Ann. of Math. 66 (1957), 1-26.

10. H. F. Trotter, Homology of group systems with application to knot theory, Ann. of Math. 76 (1962), 464-498.

Princeton University

The core $k$ of $B$ is a simple closed curve in $B$ which is homeomorphic to one of the boundary curves. 